\title{
Tetramethylpiperidine-1-oxyl-Mediated Polymerization of Styrene Using a Macroazoinitiator of Poly(ethylene oxide)
}

\author{
Eri YoshidA ${ }^{\dagger}$ and Sachiko TANIMOTO \\ Department of Polymer Science and Engineering, Kyoto Institute of Technology, \\ Goshokaido-cho, Matsugasaki, Sakyo-ku, Kyoto 606-8585, Japan
}

(Received August 14, 2000; Accepted December 8, 2000)

\begin{abstract}
The radical polymerization of styrene was performed at $130^{\circ} \mathrm{C}$ for $72 \mathrm{~h}$ using 4-methoxy-2,2,6,6tetramethylpiperidine-1-oxyl (MTEMPO) and a macroazoinitiator of poly(ethylene oxide) (PEO-AZ). It was found that a block copolymer consisting of PEO and polystyrene (PSt) was quantitatively obtained when the ratio of MTEMPO to the azo moiety in PEO-AZ was 1.0. The polymerization proceeded in accordance with a living mechanism, because the molecular weight of the copolymer and unit ratio of styrene to EO linearly increased with increasing the conversion of styrene. DSC analysis demonstrated that the copolymers had two glass transition originating from the PEO and PSt segments, and a melting endotherm based on the PEO. Furthermore, the PEO- $b$-PSt copolymer initiated the polymerization of 4-methoxystyrene, giving a block copolymer composed of PEO, PSt, and poly(4-methoxystyrene).

KEY WORDS 4-Methoxy-tetramethylpiperidine-1-oxyl / Poly(ethylene oxide) / Macroazoinitiator / Living Radical Polymerization / Initiator Efficiency / Styrene / Block Copolymer /
\end{abstract}

Considerable attention has been focused on preparing new polymer materials as a variety of properties and functions of polymers have been required. The ways of the preparation are, for instance, polymer blend, ${ }^{1,2}$ chemical and physical modification, ${ }^{3,4}$ control of molecular structure, ${ }^{5-7}$ and synthesis of new types of polymers. ${ }^{8-10}$ Synthesis of block copolymers is convenient to create new materials through combining polymers with desirable properties. A large number of block copolymers have been prepared by using a technique of sequential addition of a monomer to a polymer solution in living polymerizations. The polymerizations include anionic, cationic, and radical polymerizations. Transformation polymerization involving two or more polymerizations is useful to create new polymer materials, because it can give block copolymers in which the polymer segments are obtained from different types of polymerizations. Transformation including living radical polymerization is significant in point of the fact that a variety of monomers can be used for radical polymerization. 4Substituted - 2, 2, 6, 6 - tetramethylpiperidine - 1 - oxyl (TEMPO) serves as a counter radical for living radical polymerization, and gives polymers with narrow polydispersity. This radical also serves as a transforming agent in transformation polymerization, giving block copolymers. For instance, the synthesis of $\operatorname{poly}(\varepsilon$ caprolactone)- $b$-polystyrene (PSt) diblock copolymers was attained by the transformation from living anionic polymerization of $\varepsilon$-caprolactone to living radical polymerization of styrene, using aluminum tri(4-oxyTEMPO). ${ }^{11}$ The transformation from living cationic polymerization of THF to living radical polymerization of styrene by sodium 4-oxy-TEMPO gave poly(THF)- $b$-PSt diblock and PSt- $b$-poly(THF)- $b$-PSt triblock copolymers. ${ }^{12,13}$ We also succeeded in preparing PSt- $b$-poly(ethylene adipate)- $b$-PSt triblock copolymers through transformation from polycondensation to living radical

${ }^{\dagger}$ To whom correspondence should be addressed. polymerization with 4-hydroxy-TEMPO.$^{14}$ The TEMPOmediated radical polymerization using macroazoinititors is efficient for preparing well-defined block copolymers consisting of polymers obtained through different polymerizations. Macroazoinitiators are easily prepared by polycondensation of 4,4'-azobis(4-cyanopentanoyl)dichloride with polymeric diol or diamine. Some macroazoinitiators have already been prepared: macroazoinitiators of poly(dimethylsiloxane),${ }^{15}$ polyisobutylene, ${ }^{16}$ poly(ethylene oxide), ${ }^{17}$ polyester, ${ }^{15}$ polycarbonate, ${ }^{18}$ and polyamide. ${ }^{19}$ We have already released a publication on the synthesis of PSt- $b$-poly(dimethylsiloxane) diblock copolymers through the polymerization of styrene by 4methoxy-TEMPO (MTEMPO) using a macroazoinitiator of poly(dimethylsiloxane). The films of the copolymers was obtained with the good optical qualities of colorlessness, high transparency, and flexibility. ${ }^{20}$ Poly(ethylene oxide) (PEO) is a hydrophilic polymer. This polymer is useful for improving polymer properties because the polymer is stable and soluble in common organic solvents as compared with other hydrophilic polymers such as poly(vinyl alcohol), poly(acrylic acid), and poly(acrylamide). In this paper, we describe the convenient synthesis of a block copolymer consisting of hydrophobic PSt and hydrophilic PEO by the TEMPO-mediated polymerization using PEO macroazoinitiator.

\section{EXPERIMENTAL}

\section{Measurements}

Gel permeation chromatography (GPC) was performed with a Toso HLC-802 A instrument equipped with a Toso CP-8000 chromato processor. A combination of two PSt gel columns of Toso TSK gel $\mathrm{G} 4000 \mathrm{H}_{8}$ and $\mathrm{G} 2000 \mathrm{H}_{8}$ was used, with $\mathrm{THF}$ as the eluent at $42^{\circ} \mathrm{C}$. The molecular weight was determined by calibrating with PSt standards. UV spectra were obtained with a Shimadzu UV2500 PC UV-VIS recording spectrophotometer. ${ }^{1} \mathrm{H}$ NMR spectra were also obtained with Bruker ARX-500 and 
Varian Gemini-200 NMR spectrometers. Gas chromatography (GC) was performed with a Shimadzu GC-6 A. DSC spectra was obtained with a Mac Science DSC3100 .

\section{Materials}

PEO-AZ (azo content $=0.422 \mathrm{mmol} \mathrm{g}^{-1}$ ), which was prepared by the reaction of polyethylene glycol $\left(M_{\mathrm{n}}=\right.$ 2000) with 4,4'-azobis(4-cyanopentanoyl)dichloride following a previous method, ${ }^{15}$ was supplied from Wako Pure Chemical Industry, LTD. The pure grade PEO-AZ was used without further purification. MTEMPO was prepared by the method described previously. ${ }^{21}$ Commercial grade St was washed with an aqueous alkaline solution and water, and distilled over calcium hydride. Benzene as a solvent for the reaction of PEO-AZ with MTEMPO, was washed with conc. sulfuric acid and water, and distilled over sodium. Toluene as an internal standard in GC was also distilled over sodium.

\section{Reaction of PEO-AZ with MTEMPO}

PEO-AZ (216 mg, azo content $=0.0912 \mathrm{mmol})$, MTEMPO (17 mg, $0.0914 \mathrm{mmol}$ ) and $4 \mathrm{~mL}$ of benzene were placed in an ampule. After degassing the content, the ampule was sealed in vacuo. The reaction was carried out at $80^{\circ} \mathrm{C}$ for $14 \mathrm{~h}$. It was terminated by cooling with liquid nitrogen. The resulting solution was subjected to UV analysis to calculate the initiator efficiency, just after the reaction.

\section{Radical Polymerization of Styrene by PEO-AZ and MTEMPO}

PEO-AZ (216 mg, azo content $=0.0912 \mathrm{mmol})$, MTEMPO (17 mg, $0.0914 \mathrm{mmol})$, and $4 \mathrm{~mL}(34.9 \mathrm{mmol})$ of St were placed in an ampule. After degassing the content, the ampule was sealed in vacuo. The polymerization was carried out at $130^{\circ} \mathrm{C}$ for $72 \mathrm{~h}$. It was terminated by cooling with liquid nitrogen. The product was dissolved in $10 \mathrm{~mL}$ of dichloromethane, purified by repeated precipitations from dichloromethane into methanol, and finally freeze-dried with benzene. The block copolymer of $3.68 \mathrm{~g}$ was obtained. After addition of toluene as an internal standard, the dichloromethane solution was subjected to GC to estimate the conversion of St.

\section{Reaction of 4-Methoxystyrene by PEO-b-Polystyrene}

PEO- $b$-PSt ( $\left.100 \mathrm{mg}, M_{\mathrm{n}}=49000, M_{\mathrm{w}} / M_{\mathrm{n}}=1.48\right)$ and 1 $\mathrm{mL}(7.52 \mathrm{mmol})$ of 4-methoxystyrene were placed in an ampule. After degassing the content, the ampule was sealed in vacuo. The reaction was carried out at $130^{\circ} \mathrm{C}$ for $5.5 \mathrm{~h}$. It was terminated by cooling with liquid nitrogen. The product was dissolved in $10 \mathrm{~mL}$ of dichloromethane, purified by repeated precipitations from dichloromethane into methanol, and finally freeze-dried with benzene. The block copolymer of $810 \mathrm{mg}$ was obtained. After addition of toluene as an internal standard, the dichloromethane solution was subjected to GC to estimate the conversion of 4-methoxystyrene.

\section{RESULTS AND DISCUSSION}

In the TEMPO-mediated polymerization an excess of TEMPO to the initiating radicals induces autopolymeri-
Table I. Initiator efficiency of PEO macroazoinitiator

\begin{tabular}{ccrc}
\hline \multirow{2}{*}{ MTEMPO/AZ } & $\begin{array}{c}\text { Initiator } \\
\text { efficiency }\end{array}$ & $M_{\mathrm{n}}{ }^{\mathrm{a}}$ & Proportion \\
\cline { 4 - 4 } 0.6 & - & 13000 & 0.283 \\
& & 9800 & 0.229 \\
\multirow{2}{*}{1.0} & \multirow{2}{*}{0.44} & 4000 & 0.488 \\
& \multirow{2}{*}{2.0} & 8100 & 0.290 \\
& 0.46 & 3500 & 0.710 \\
& & 8400 & 0.307 \\
\hline
\end{tabular}

a Estimated by GPC based on polystyrene standards.

zation, so that equimolar addition of TEMPO to the initiating radical is required. The initiator efficiency of $\mathrm{PEO}$ macroazoinitiator (PEO-AZ) was explored. TEMPO has a typical red color originating from $\mathrm{N}-\mathrm{O} \cdot$ and shows $\mathrm{UV}$ absorbance at $469 \mathrm{~nm}$ as $\lambda_{\text {max }}$. This radical captures carbon radical giving the alkoxyamine, which has no UV absorbance. The initiator efficiency is assumed to be the efficiency with which TEMPO captures the initiating radical, and can be determined by UV at $469 \mathrm{~nm}$. The reaction of PEO-AZ and MTEMPO was carried out in benzene at $80^{\circ} \mathrm{C}$ for $14 \mathrm{~h}$. The results are shown in Table I. When a ratio of MTEMPO to azo moiety (MTEMPO/AZ) in PEO-AZ was 0.6, no absorbance of MTEMPO was observed, after the reaction. This indicates that the amount of MTEMPO was not enough to capture all the initiating radicals. In the cases of MTEMPO/AZ $\geqq 1$, observation of the UV absorbance was made due to MTEMPO, even after the reaction. The initiator efficiency was estimated to be $0.44-0.46$. $\mathrm{PEO}-\mathrm{AZ}$ was prepared by polycondensation of $4,4^{\prime}$-azobis ( 4cyanopentanoyl)dichloride and poly(ethylene glycol) having a molecular weight of 2000 . The macroazoinitiator has a molecular weight $\left(M_{\mathrm{n}}\right)$ of 30000 . Therefore, 13 azo moieties and 14 PEO segments are contained in a molecule of the macroazoinitiator. It was found that the reaction gave two pieces of $\mathrm{PEO}$ products: one is an oligomer with $M_{\mathrm{n}}=3500-3800$ and the other is a polymer having $M_{\mathrm{n}}=8100-8400$. These molecular weights were estimated by GPC based on PSt standards. The ratio of the oligomer to the polymer produced was oligomer: polymer $=c a .0 .70: 0.30$, based on the molecular weights and their area percents in GPC. This suggests that two types of PEO blocks were generated: one is the PEO block generated by two PEO segments combining. The other is the block comprising from four PEO segments bound. These results indicate that four types of initiating radicals are generated from the $\mathrm{PEO}$ macroazoinitiator (Scheme 1). The initiator efficiency of PEO-AZ is a little higher than that of a macroazoinitiator of poly(dimethylsiloxane) (PDMS). ${ }^{20}$ This can be accounted for by the fact that PDMS-AZ had longer PDMS segments $\left(M_{\mathrm{n}}\right.$ $=5000$ ) and lower azo content.

The bulk polymerization of St was performed for $72 \mathrm{~h}$ at $130^{\circ} \mathrm{C}$ under the condition of MTEMPO/AZ $=1.0$ (Scheme 2). The product was isolated and purified through repeated reprecipitation into methanol. The methanol solution obtained after the precipitation was evaporated to remove the methanol, with the result that no residue was yielded. This result indicates that no homopolymer of PEO was generated through the polymeri- 


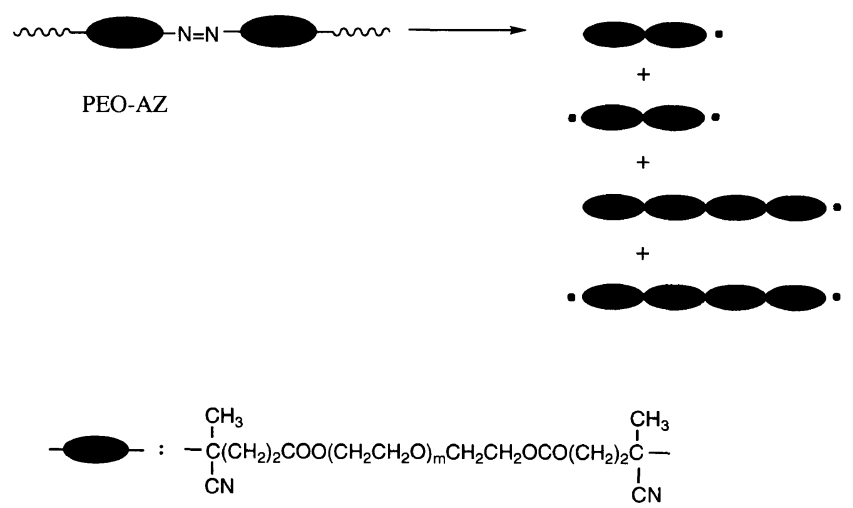

Table II. The radical polymerization of styrene by PEO-AZ and MTEMPO

\begin{tabular}{ccrc}
\hline MTEMPO/AZ & Conversion $/ \%$ & \multicolumn{1}{c}{$M_{\mathrm{n}}{ }^{\mathrm{b}}$} & $M_{\mathrm{w}} / M_{\mathrm{n}}{ }^{\mathrm{b}}$ \\
\hline 0.4 & 100 & 57000 & 2.17 \\
0.6 & 99 & 120000 & 1.43 \\
0.8 & 94 & 79000 & 1.54 \\
1.0 & 95 & 72000 & 1.61 \\
1.5 & 93 & 27000 & 1.34 \\
2.0 & 100 & 26000 & 1.88 \\
4.0 & 100 & 21000 & 1.49 \\
\hline
\end{tabular}

${ }^{a}$ Estimated by GC. ${ }^{\mathrm{b}}$ Estimated by GPC based on polystyrene standards.

Scheme 1.

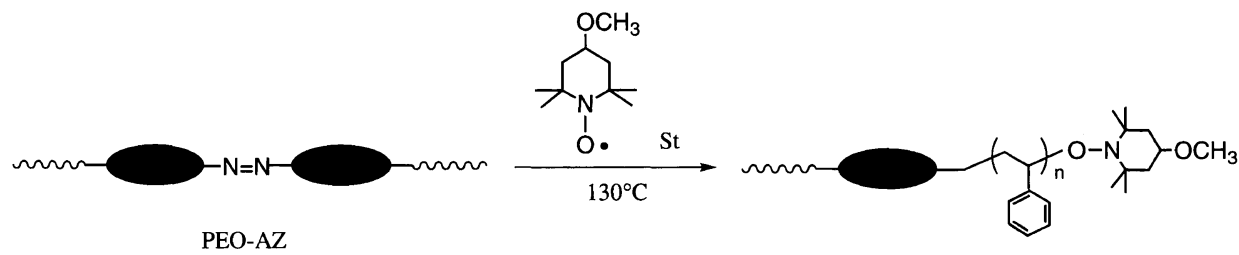

Scheme 2.

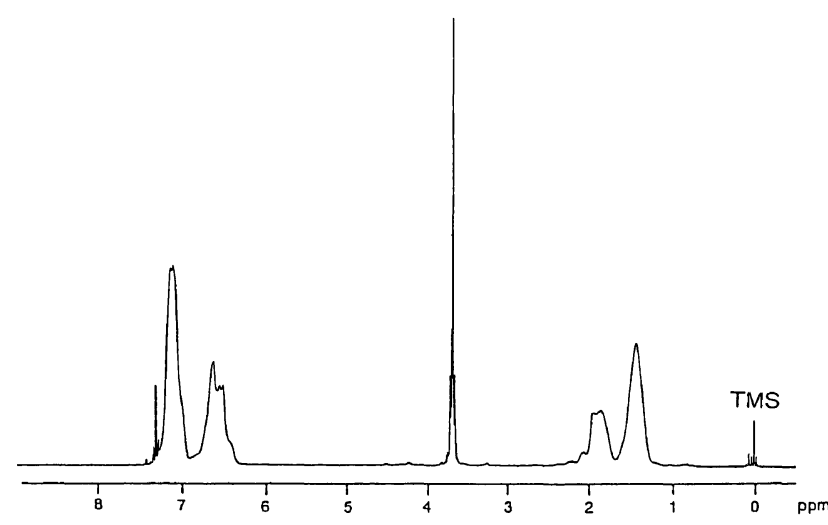

Figure 1. ${ }^{1} \mathrm{H}$ NMR spectrum of the copolymer obtained by the radical polymerization of styrene by PEO-AZ and MTEMPO (solvent: $\mathrm{CDCl}_{3}$ ).

zation. All the PEO segments engaged in the polymerization to form the block copolymer consisting of PEO and PSt segments. The copolymer showed 72000 as a molecular weight and 1.61 as polydispersity. Figure 1 shows ${ }^{1} \mathrm{H}$ NMR spectrum of the copolymer. While signals due to PSt blocks were observed 1.2-2.2 and 6.3$7.3 \mathrm{ppm}$, signals originating from PEO blocks were discerned at $3.72 \mathrm{ppm}$. The unit ratio of St to EO was estimated to be 9.07. The relationship between MTEMPO/ $\mathrm{AZ}$, the conversion, molecular weight, and polydispersity is summarized in Table II. The molecular weight decreased with an increase of MTEMPO/AZ, while most of the polydispersities were 1.6 or less, with the exception of $\mathrm{MTEMPO} / \mathrm{AZ}=0.4$. Ueda and his co-workers reported on the polymerization of St by PEO-AZ in the absence of TEMPO. ${ }^{17}$ The uncontrolled polymerization gave copolymers having polydispersities of 1.9-4.5 when the molecular weights were over 10000 . It was found that the presence of TEMPO made a significant contribution to narrowing polydispersity of the copolymers. GPC pro-

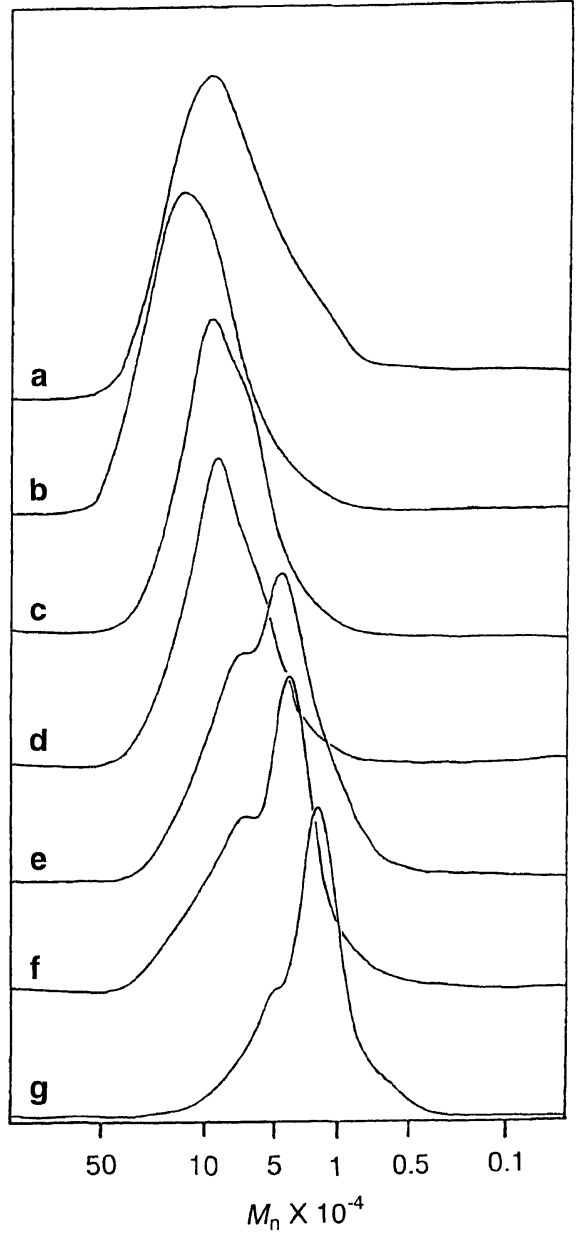

Figure 2. GPC profiles of the block copolymers obtained from the polymerization of styrene by PEO-AZ and MTEMPO: MTEMPO/ $\mathrm{AZ}=0.4(\mathbf{a}), 0.6(\mathbf{b}), 0.8(\mathbf{c}), 1.0(\mathbf{d}), 1.5(\mathbf{e}), 2.0(\mathbf{f})$, and $4.0(\mathbf{g})$.

files of the copolymers obtained by the MTEMPOmediated polymerization are shown in Figure 2. When 


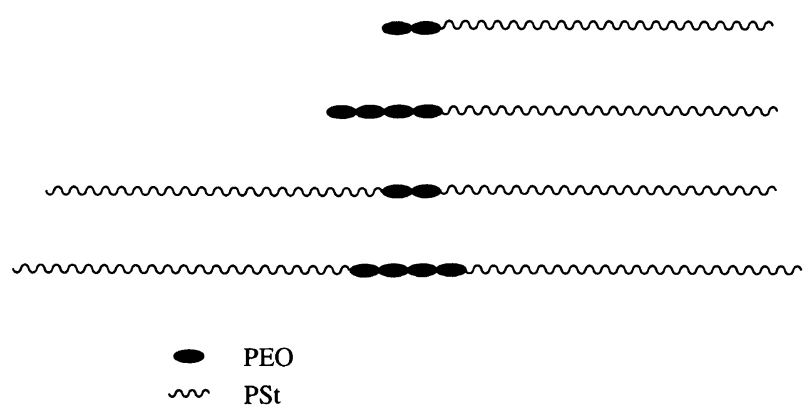

Scheme 3.

Table III. Relation of molecular weight, polydispersity, and unit molar ratio to conversion

\begin{tabular}{|c|c|c|c|c|}
\hline$\frac{\text { Time }}{\mathrm{h}}$ & $\frac{\text { Conversion }^{\mathrm{a}}}{\%}$ & $M_{\mathrm{n}}^{\mathrm{b}}$ & $M_{\mathrm{w}} / M_{\mathrm{n}}^{\mathrm{b}}$ & $\mathrm{St} / \mathrm{EO}$ \\
\hline 1 & 35 & 20000 & 1.39 & 1.73 \\
\hline 2 & 38 & 28000 & 1.47 & 2.51 \\
\hline 6 & 62 & 45000 & 1.43 & 5.60 \\
\hline 12 & 69 & 49000 & 1.48 & 6.40 \\
\hline 72 & 95 & 72000 & 1.61 & 9.07 \\
\hline
\end{tabular}

a Estimated by GC. ${ }^{\mathrm{b}}$ Estimated by GPC based on polystyrene standards.

MTEMPO/AZ was below 0.8, broad curves were observed. This indicates that the amount of MTEMPO was not enough to control all the growing chain ends generated from the initiating radicals, and that uncontrolled free radical polymerization competitively proceeded with the TEMPO-controlled polymerization. On the other hand, observation of sharp but bimodal GPCs was made when MTEMPO/AZ was over 1.0. The sharp main peak shifted to lower molecular weight side as the ratio of MTEMPO/AZ increased. This implies that an excess of MTEMPO induces autopolymerization of styrene, and produces St homopolymer. It is suitable that MTEMPO/ $\mathrm{AZ}$ is unity to prepare the block copolymer quantitatively. This result is in a good agreement with the initiator efficiency of PEO-AZ. A shoulder peak was discerned in GPC even when MTEMPO/AZ was unity. This suggests that the product is a mixture of PEO- $b$-polystyrene diblock and polystyrene- $b$-PEO- $b$-polystyrene triblock copolymers. Based on the analysis of the initiator efficiency, it is expected that the product consists of two types of diblock copolymers and also two types of triblock copolymers (Scheme 3). It should be difficult to distinguish the two types of diblock copolymers or the two types of triblock copolymes by GPC, because PSt blocks are quite longer than PEO blocks.

We investigated whether the polymerization of St under the condition that MTEMPO/AZ was unity. The results are shown in Table III. The conversion, molecular weight, and unit ratio of St to EO increased over time. All the polydispersities were completely narrow and less than 1.5. Figure 3 shows the first-order time-conversion plots in the polymerization. The $\ln [\mathrm{M}]_{0} /[\mathrm{M}]$ is proportional to time, however, it increased over time curvilinearly rather than linearly. This may be accounted for because the initiating radicals don't initiate St polymerization at the same time, due to the high diffusion ratedetermining step of the macroazoinitiator. On the other

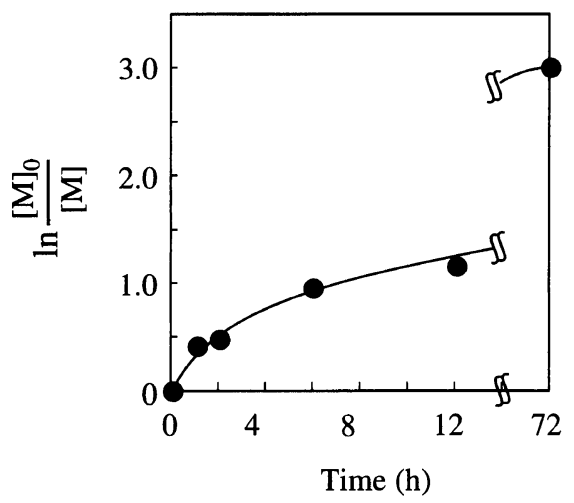

Figure 3. First order time-conversion plots in the polymerization of styrene by PEO-AZ and MTEMPO where the ratio of MTEMPO to the azo moiety was 1.0 .

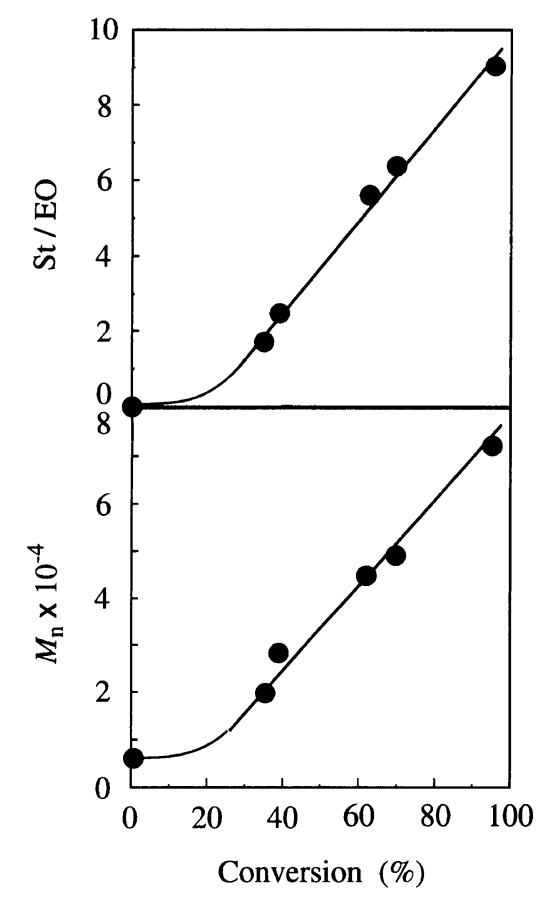

Figure 4. Plots of the molecular weight and molar ratio of styrene to EO units in the copolymer $v s$. conversion for the polymerization of styrene by PEO-AZ and MTEMPO where MTEMPO/AZ= 1.0 .

hand, the molecular weights of the copolymers and the unit ratios of St to EO linearly increased with an increase of the conversion (Figure 4). These results indicate that the polymerization proceeded in accordance with a living mechanism under the condition of $\mathrm{MTEMPO} / \mathrm{AZ}=1.0$.

Hydrophobic PSt is expected to be completely immiscible with hydrophilic PEO. DSC analysis demonstrated that the copolymer had two glass transition originating from PSt blocks and PEO. Figure 5 shows DSC curves for St homopolymer, PEO-AZ, and the copolymers. The PSt with $M_{\mathrm{n}}=14000$ and $M_{\mathrm{w}} / M_{\mathrm{n}}=1.53$ had the glass transition at $91^{\circ} \mathrm{C}$. PEO-AZ showed two peaks in the DSC: an exotherm at $-45^{\circ} \mathrm{C}$ and a melting endotherm at $33^{\circ} \mathrm{C}$. The exotherm originates from cold crystallization of the amorphous PEO, indicating that the azo moieties attached to the PEO segments prevent the PEO blocks 


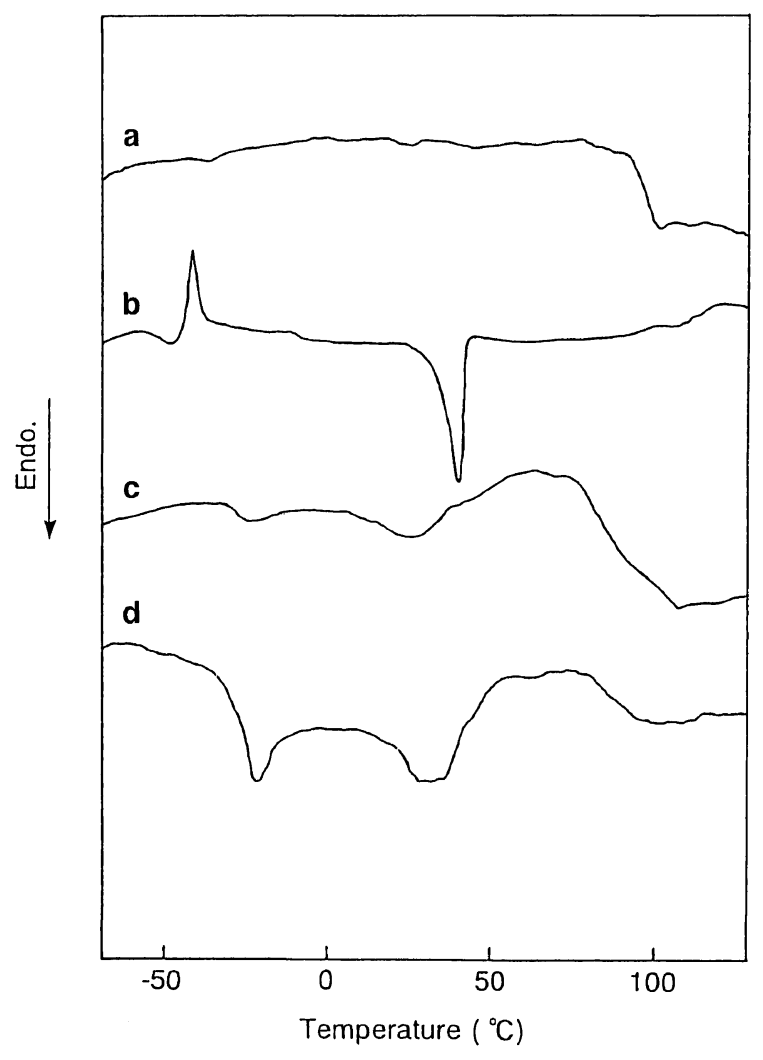

Figure 5. DSC curves of a styrene homopolymer $\left(\mathbf{a}, M_{\mathrm{n}}=14000\right.$, $\left.M_{\mathrm{w}} / M_{\mathrm{n}}=1.53\right)$, PEO-AZ (b), and the copolymers (c, $M_{\mathrm{n}}=20000$, $M_{\mathrm{w}} / M_{\mathrm{n}}=1.39$, and d, $M_{\mathrm{n}}=72000, M_{\mathrm{w}} / M_{\mathrm{n}}=1.61$ ) obtained from the polymerization of styrene by PEO-AZ and MTEMPO where MTEMPO/AZ $=1.0$.

from crystallizing. The copolymers had two glass transition attributed to $\mathrm{PEO}$ and polystyrene blocks and a melting exotherm. While the copolymer with a unit ratio of St to EO (St/EO) of $1.73\left(M_{\mathrm{n}}=20000\right.$ and $M_{\mathrm{w}} / M_{\mathrm{n}}=$ 1.39) showed these three peaks at $-30,75$, and $15^{\circ} \mathrm{C}$, the copolymer with $\mathrm{St} / \mathrm{EO}=9.07\left(M_{\mathrm{n}}=72000\right.$ and $M_{\mathrm{w}} / M_{\mathrm{n}}$ $=1.61$ ) had the peaks at $-34,80$, and $18^{\circ} \mathrm{C}$, respectively. The former copolymer showed smaller intensities of the peaks due to the $\mathrm{PEO}$ blocks $\left(-30\right.$ and $\left.15^{\circ} \mathrm{C}\right)$ than the latter $\left(-34\right.$ and $18^{\circ} \mathrm{C}$ ), in spite that the former had shorter PSt segments than the later. This implies that aggregation of the PEO blocks is promoted as a ratio of the immiscible PSt segments increase.

The PEO- $b$-PSt was found to initiate the radical polymerization of 4-methoxystyrene (MSt). The polymerization was performed at $130^{\circ} \mathrm{C}$ for $5.5 \mathrm{~h}$ using PEO- $b$ PSt (Table III, $M_{\mathrm{n}}=49000$ and $M_{\mathrm{w}} / M_{\mathrm{n}}=1.48$ ). Figure 6 shows GPC profiles of the copolymer obtained and the PEO- $b$-PSt prepolymer. The copolymer produced is also expected to consist of 4 types of copolymers, based on the structure of the PEO- $b$-PSt prepolymer (Scheme 4). The conversion of MSt was $99 \%$. The PEO- $b$-PSt- $b$-PMSt had $M_{\mathrm{n}}=120000$ and $M_{\mathrm{w}} / M_{\mathrm{n}}=1.53$. It is hard in GPC to observe a peak of the PEO- $b$-PSt prepolymer in the copolymer produced, suggesting that the copolymer efficiently initiated the polymerization of MSt, giving the triblock copolymer.

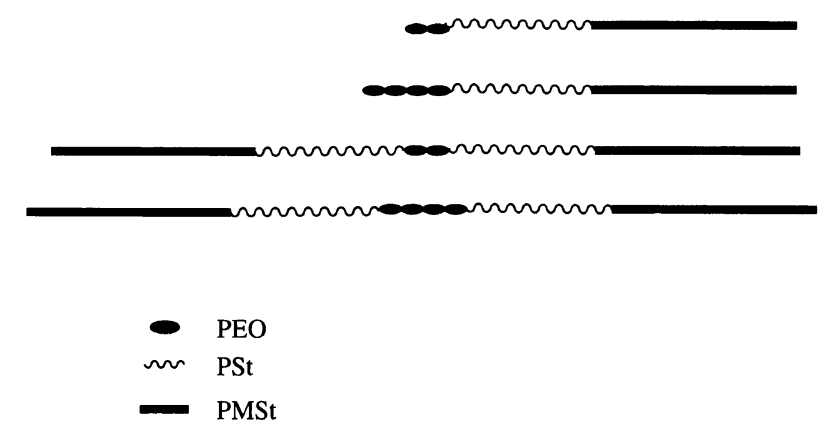

Scheme 4.

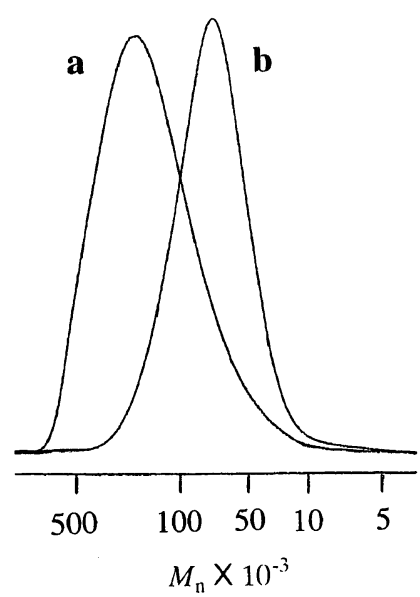

Figure 6. GPC profiles of the copolymer produced (a) and the PEO-b-PSt prepolymer (b).

\section{REFERENCES}

1. D. R. Paul and J. W. Barlow, J. Macromol. Sci. Rev. Macro mol. Chem., 18, 109 (1980).

2. O. Olabisi, L. M. Robeson, and M. T. Shaw, "Polymer-Polymer Miscibility”, Academic Press, Inc., New York, N.Y., 1979.

3. D. C. Sherrington and P. Hodge, Ed., "Syntheses and Separations Using Functional Polymers", John Wiley \& Sons, Ltd., Chichester, UK, 1988.

4. S. Wu, "Polymer Interfaces Adhesion", Marcel Dekker, Inc., New York, N.Y., 1982.

5. M. Szwarc, Nature, 178, 1168 (1956).

6. S. Smith and A. Hubin, J. Macromol. Sci., Chem., A 7, 1399 (1973).

7. T. E. Patten, J. Xia, T. Abernathy, and K. Matyjaszewski, Science, 272, 866 (1996).

8. K. Aoi, K. Itoh, and M. Okada, Macromolecules, 28, 5391 (1995).

9. Y. X. Shen and H. W. Gibson, Macromolecules, 25, 2058 (1992).

10. D. Greszta and K. Matyjaszewski, Polym. Prepr. (Am. Chem. Soc. Div. Polym. Chem.), 37, 569 (1996).

11. E. Yoshida and Y. Osagawa, Macromolecules, 31, 1446 (1998).

12. E. Yoshida and A. Sugita, Macromolecules, 29, 6422 (1996).

13. E. Yoshida and A. Sugita, J. Polym. Sci., PartA: Polym. Chem., 36, 2059 (1998).

14. E. Yoshida and M. Nakamura, Polym. J., 30, 915 (1998).

15. H. Inoue, A. Ueda, and S. Nagai, J. Polym. Sci., Polym. Chem., 26, 1077 (1988).

16. M. K. Mishra, Macromolecules, 29, 5228 (1996).

17. A. Ueda and S. Nagai, J. Polym. Sci., Polym. Chem., 24, 405 (1986).

18. M. Shimada, Y. Uetoshi, and Y. Makimura, Polym. Prepr. 
Jpn., 8, 1958 (1996).

19. A. Ueda and S. Nagai, J. Polym. Sci., Polym. Chem., 22, 1611 (1984).

20. E. Yoshida and S. Tanimoto, Macromolecules, 30, 4018
(1997).

21. T. Miyazawa, T. Endo, S. Shiihashi, and M. Okawara, J. Org. Chem., 50, 1332 (1985). 